\title{
Energieeffiziente Gebäude als Schlüssel für eine nachhaltige Entwicklung
}

Rund ein Drittel der weltweit energiebezogenen $\mathrm{CO}_{2}$-Emissionen sind auf den Verbrauch durch Gebäude zurückzuführen. Ohne eine sogenannte Gebäudewende sind die notwendigen $\mathrm{CO}_{2}-$ Einsparungen nicht zu erreichen. Eine Internetplattform bündelt das weit verstreute Wissen zu diesem Thema und bietet Orientierung. Von Jana Rasch, Kilian Topp und Stefan Thomas

O bwohl Energieeffizienz erfolgreich auf dem Weg zur ersten Energiequelle ist und die globalen Investitionen in Energieeffizienz in 2014 höher ausfielen als die Investitionen in die Elektrizitätserzeugung durch erneuerbare Energien, Kohle, Gas und Öl, bleiben weiterhin große Potenziale ungenutzt (IEA 2014). Das gilt insbesondere für den Gebäudesektor, der bereits aufgrund seiner Größe und seiner Einsparpotenziale besonderen Augenmerks bedarf.

Das Energieszenario des Global Energy Assessment Reports bestätigt, dass durch den Einsatz modernster energieeffizienter Bau- und Sanierungslösungen bis $205046 \%$ des globalen Heiz- und Kühlendenergieverbrauchs, im Vergleich zum Referenzjahr 2005, eingespart werden können (GEA 2012). Die Lücke in der Umsetzung im Vergleich zum Trendfall, mit einer Zunahme des Heiz- und Kühlenergieverbrauchs um $33 \%$, beträgt im Jahr 2050 sogar insgesamt $79 \%$ (siehe Abbildung 1).

Diese Umsetzungslücke zu schließen, stellt eine große Herausforderung dar, der sich die politischen Entscheidungsträger, die Aufsichtsbehörden und relevanten Marktteilnehmer stellen müssen. Eine umfassende Übersicht über Technologien, Politikansätze und GoodPractice-Beispiele zu erstellen und die vielfältigen Einzelteile zu einem Gesamtbild zusammenzutragen, ist eine ebenso ambitionierte wie mühsame Arbeit. Als Hilfestellung und Katalysator für einen globalen Wissenstransfer im Bereich Gebäudeenergie ist sie jedoch sehr vielversprechend.

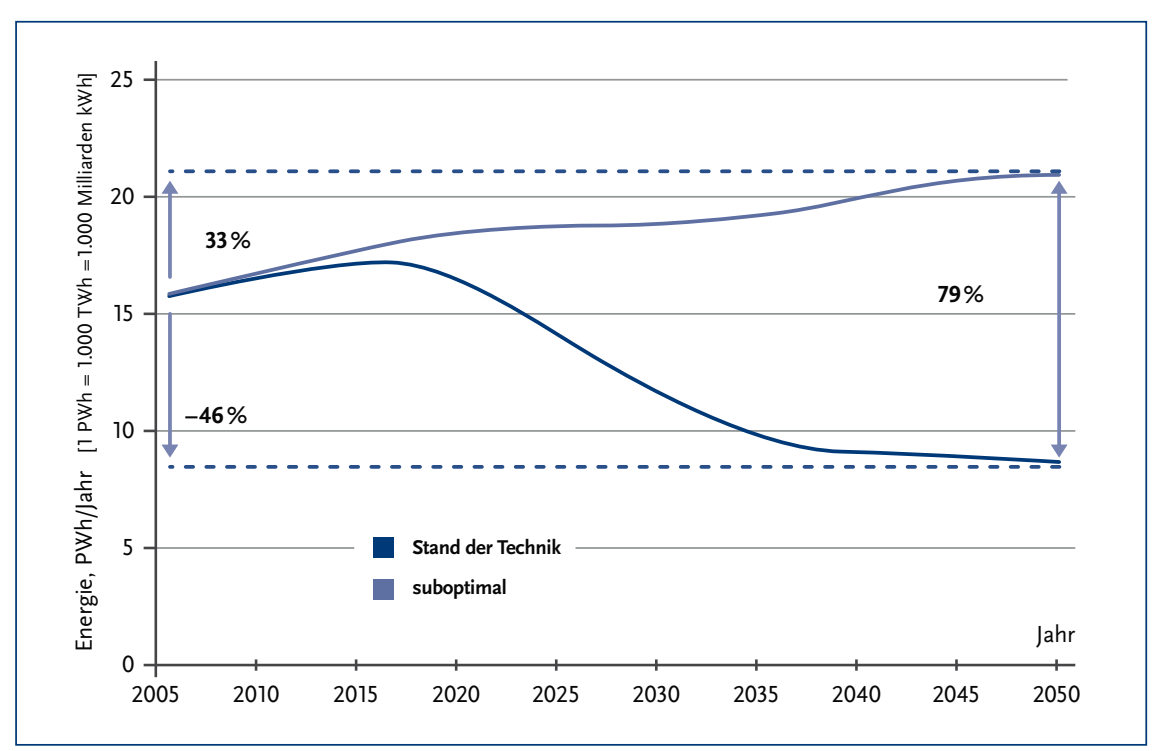

Abbildung 1: Weltweiter Raumwärme- und Raumkälteendenergieverbrauch 2005 bis 2050, suboptimales und modernstes Energieeffizienzszenario

\section{Weltweiter Wissenstransfer}

Informationen zu Technologien und Politiken existieren in vielfältiger Form. Allerdings sind diese meist verstreut und auch für Entscheidungsträger schwer zugänglich. Vor allem für Akteure in Entwicklungs- und Schwellenländern stellt dies ein Problem dar. Wissen zu Gebäudekonzepten, Potenzialen und guten Beispielen ist dort oft nur lückenhaft vorhanden und die vorhandenen Informationen sind teils widersprüchlich. Dieser Mangel ist umso dringender zu beheben, da dieses Wissen für Entwicklungs- und Schwellenländer aufgrund des anhaltenden Wachstums von Gebäudefläche, Energie- und Ressourcenverbrauch für die Entwicklungs- und Klimaschutzziele eine bedeutende Rolle spielt.

Das Projekt bigEE adressiert dieses Problem, indem Wissen gebündelt wird und verständliche, unabhängige und umfassende Informationen zur Energieeffizienz in Gebäuden zur Verfügung gestellt werden, um politische wie Investitionsentscheidungen und deren Umsetzung zu erleichtern. Ziel der Initiative ist es, einen Beitrag zum globalen Wissensmanagement und Wissenstransfer zu leisten. Insgesamt verfolgt bigEE dabei die folgenden Ziele:

I Förderung des Bewusstseins für die Vorteile von verbesserter Energieeffizienz bestehender und neuer Gebäude. 
- Bündelung von Informationen und Materialien zu den weltweiten Chancen für Energieeffizienz, insbesondere in Schwellen- und Entwicklungsländern.

- Wissensmanagement und Kommunikation vorhandener Informationen, indem Wissen zielgruppenorientiert, konsistent, verständlich, transparent und leicht zugänglich zur Verfügung gestellt und aktualisiert wird.

\section{Energieeffiziente Gebäude und passendes Politikpaket}

Um diese Ziele zu erreichen, wurde bigEE als eine internationale, durch das Wuppertal Institut koordinierte, Initiative von Forschungsinstituten und öffentlichen Behörden im Bereich Energie und Klima gegründet. Im Zentrum steht

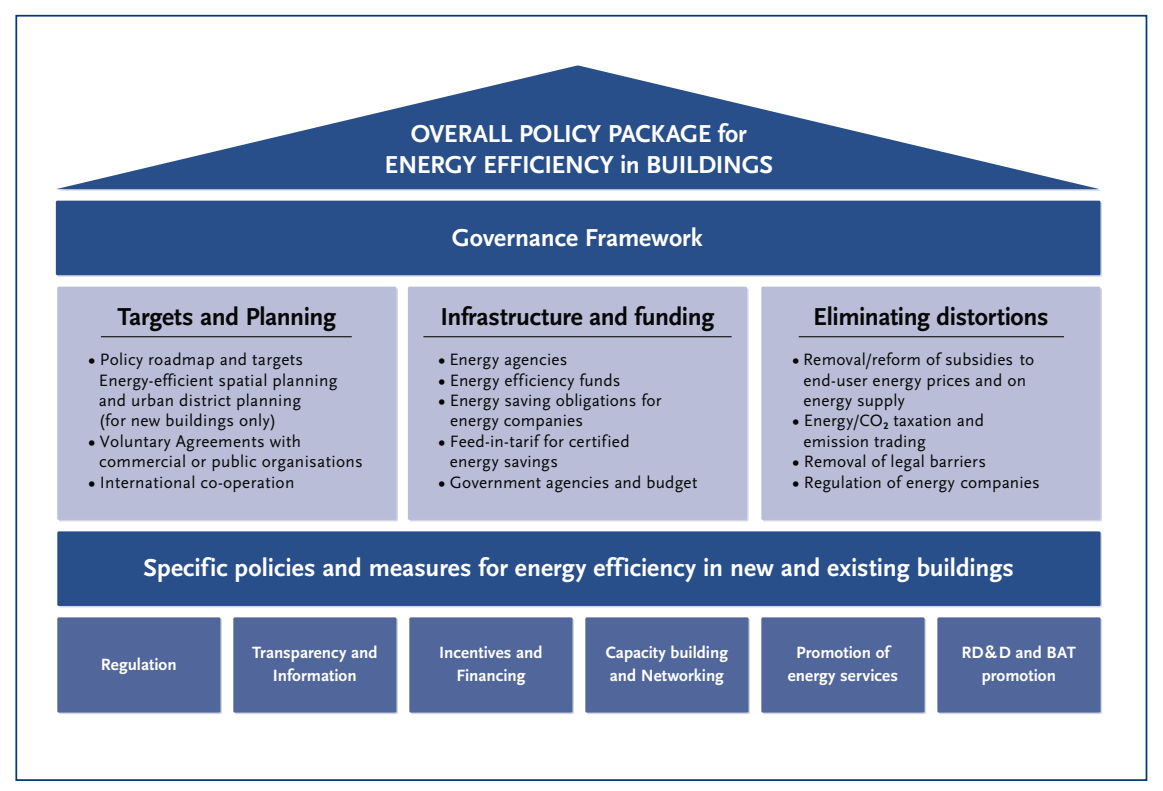

Abbildung 2: Elemente des empfohlenen Politikpakets für Energieeffizienz in neuen Gebäuden

(eigene Darstellung) die web-basierte Wissensplattform www.

bigee.net, die Informationen zu Energieeffizienz in Gebäuden, gebäudebezogenen Technologien und Geräten - differenziert nach den Hauptklimazonen der Welt - bietet und zudem Politikempfehlungen zur Hebung der vorhandenen Potenziale zur Verfügung stellt. Die Plattform informiert über Möglichkeiten zu Energieeffizienz und Einsparungen, benennt Vorteile für Investoren und zeigt der Politik Wege auf, die die Nutzung der Potenziale befördert. Gezielte Informationen werden mit bewährten Beispielen aus der Praxis gekoppelt. Um zielgruppengerechte, schnelle und einfach zugängliche Informationen über Gebäude, Geräte und Politikmaßnahmen zu gewährleisten, gibt es drei themenspezifische Rubriken, die sogenannten Guides:

- Policy Guide,

- Buildings Guide,

- Appliances Guide.

Für die Erreichung der genannten Ziele visiert bigEE eine integrierte Herangehensweise mit der Verknüpfung von Politik und Technologie an. Im Nachfolgenden sollen die strategischen Ansätze in den Bereichen Politik und Gebäude sowie ihre Verknüpfung erläutert werden.

Wie die Ergebnisse des Projekts zeigen, erfordert die Nutzung der Energieeffizienzpotenziale, die zahlreichen Hemmnisse für die verschiedenen Marktakteure (Sorrell et al. 2004; Thomas 2007) durch die Anwendung eines umfangreichen Politikpakets mit verpflichtenden und freiwilligen Elementen $\mathrm{zu}$ überwinden (Wuppertal Institut 2012a; Thomas et al. 2013). Abbildung 2 zeigt die Komponenten des empfohlenen Politikpakets. Eine klare Vision und Ziele für Energieeffizienz sollten als Rahmen auf höchster Regierungsebene etabliert werden. Dies muss einhergehen mit der Zuteilung von Finanzmitteln und Ressourcen für die Implementierung von sektoralen Politiken und gleichzeitig Unvollkommenheiten der Märkte ad- ressieren. Auf einem sektorspezifischen Niveau sind Politikinstrumente wie Vorschriften, Anreize und Finanzierung sowie Kapazitätsaufbau wichtige Komponenten eines umfangreichen Politikpakets für Energieeffizienz in Gebäuden. Die Politikpakete wurden im Einzelnen ausführlich aufbereitet und auf der Plattform zur Verfügung gestellt, zusammen mit GoodPractice-Beispielen.

Die Abbildungen 3 und 4 zeigen, wie sektorspezifische Politikinstrumente interagieren, sich gegenseitig stärken und so den Markt für Neubauten und die energetische Sanierung von Bestandsgebäuden hin zu einer höheren Energieeffizienz transformieren.

Im Bereich Neubauten ist es Aufgabe der Politik, den Markt allmählich hin zum höchstmöglichen Niveau der Gesamtenergieeffizienz zu bewegen. Schließlich sollen auf diesem Wege Passivhäuser beziehungsweise Ultra-Low-Energy Buildings (ULEB) zum Standard werden. Dieses Ziel kann mit der Kombination von Energetischen Mindeststandards, den Minimum Energy Performance Standards (MEPS) und den übrigen in Abbildung 3 gezeigten Instrumenten zur Markteinführung von ULEB erreicht werden. Für die Sanierung bestehender Gebäude gilt ein zweifaches Ziel. Erstens sollen durch die Sanierung möglichst hohe Effizienzstandards erreicht werden, wobei deren exakte Höhe von den Klimazielen abhängig ist (siehe dazu die vertikale Achse in Abbildung 4). Zweitens muss ein entsprechend hoher Anteil des Gebäudebestandes jährlich saniert werden (siehe dazu die horizontale Achse in Abbildung 4).

Auf der vertikalen Achse ist somit die Sanierungstiefe, auf der horizontalen Achse die Sanierungsrate abgetragen. Die im Rahmen von bigEE vorgeschlagenen Politikpakete ermöglichen sowohl eine hohe Sanierungstiefe als auch hohe Sanierungsra- 


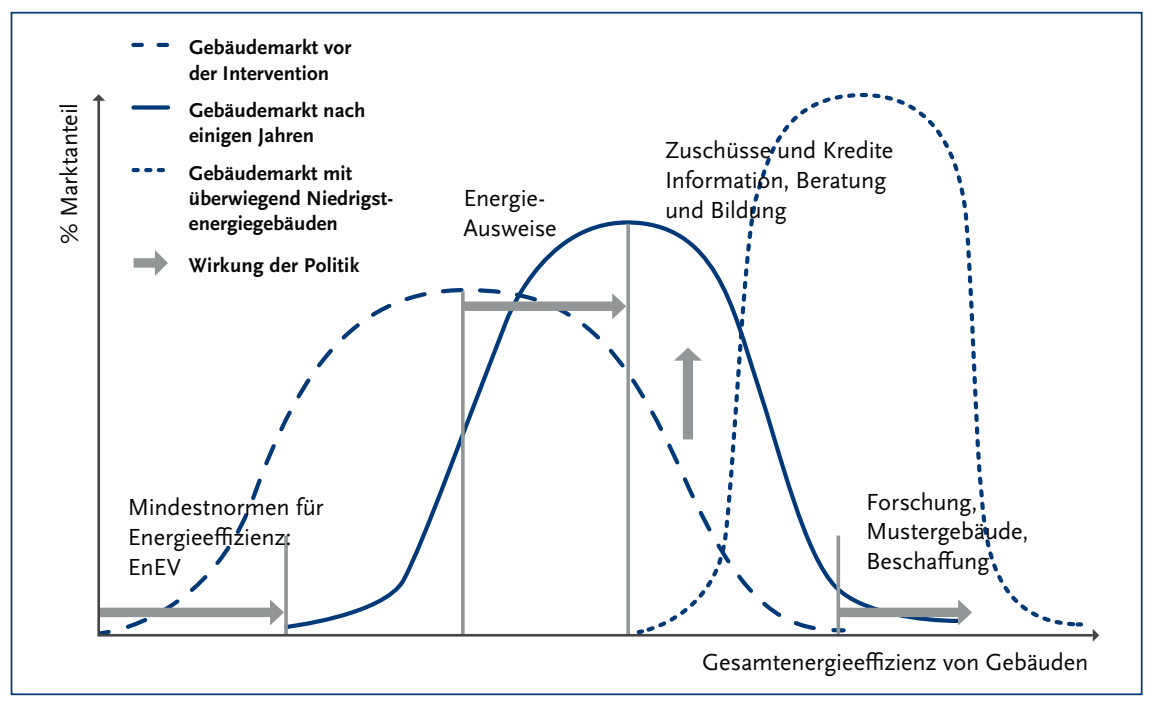

Abbildung 3: Interaktionen von Politikinstrumenten für Energieeffizienz in neuen Gebäuden (Wuppertal Institut 2012a, basierend auf Klinckenberg Consultants 2006)

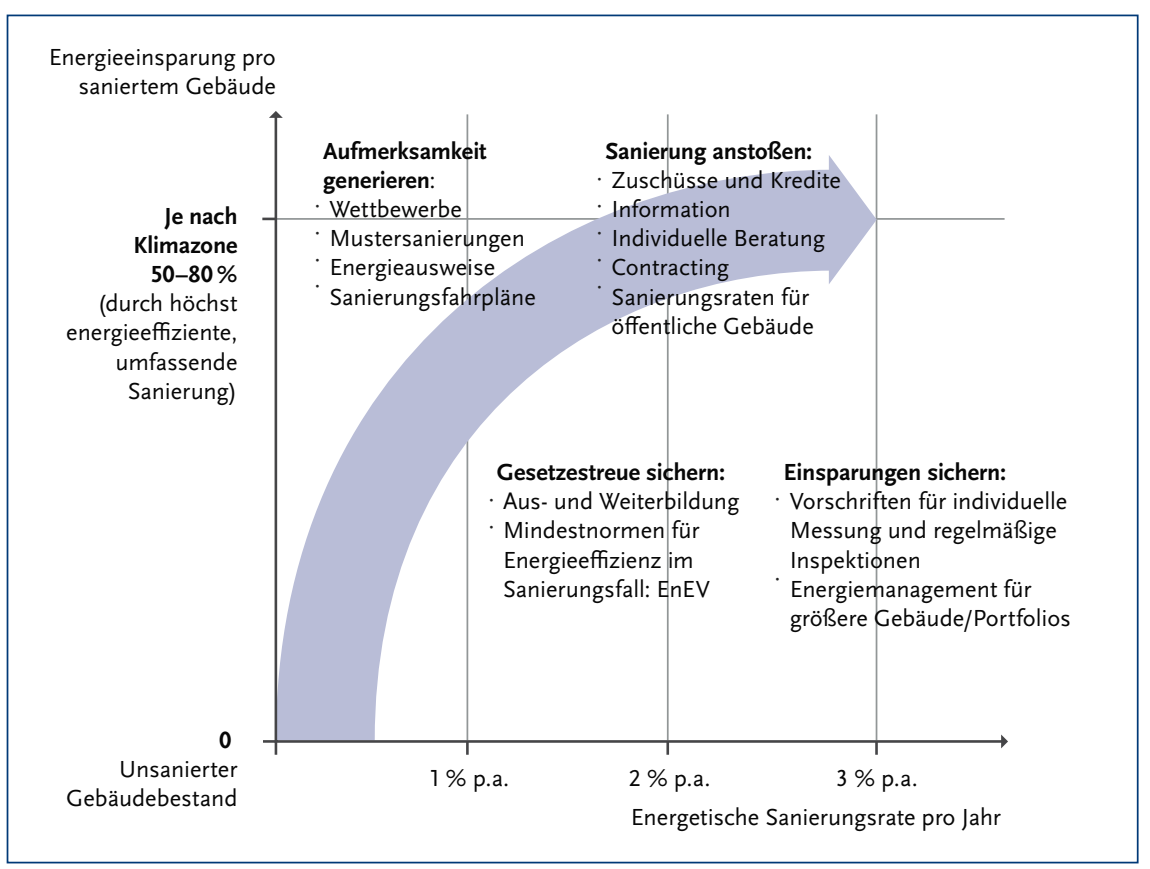

Abbildung 4: Interaktionen von Politikinstrumenten für Energieeffizienz in sanierten Gebäuden und deren Betrieb

(Wuppertal Institut 2012a)
- Detaillierte Beschreibungen der einzelnen Elemente des Politikpakets (im Internet unter: http://www.bigee .net/en/policy/guide/buildings/ package-elements)

- Praxisbeispiele für Politikpakete aus verschiedenen Ländern (im Internet unter: http://www.bigee.net/en/ policy/guide/buildings/package_examples)

- Praxisbeispiele für einzelne Politikinstrumente aus verschiedenen Ländern (im Internet unter: http://www. bigee.net/en/policy/guide/buildings/ policy/examples)

Es ist zweckmäßig, dass förderliche Rahmenbedingungen und einzelne Politikmaßnahmen an regionale Gegebenheiten angepasst und miteinander kombiniert werden, um regionsspezifische Hemmnisse für den Ausbau von Energieeffizienz $\mathrm{zu}$ überwinden und relevante Akteure erfolgreich einzubinden. Aufgrund der unterschiedlichen lokalen Rahmenbedingungen wie Klimazonen, Entwicklungsniveau oder Verfügbarkeit von Technologien ist in vielen Ländern kurz- bis mittelfristig nur eine stufenweise Annäherung an einen Hocheffizienzstandard (ULEB) möglich. Dazu wurden verschiedene Konzepte entwickelt.

Ein strategischer Ansatz für ein integriertes Gebäudedesign ist der Schlüssel, um hohe Energieeinsparungen bei geringen oder ohne zusätzliche Investitionskosten zu erreichen. Energieeffiziente Gebäude werden durch ein ganzheitliches Design mit energieeffizienten Technologien und intelligentem Gebäudemanagement ermöglicht. Beispiele für gebaute ULEB sowie Nullenergie- und Plusenergiegebäude existieren bereits. Diese haben gezeigt, dass die Erreichung dieser Ziele wirtschaftlich möglich ist. Eine

ten, indem sie erstens Aufmerksamkeit generieren, zweitens Anreize für Sanierungen bieten, drittens die Einhaltung von Standards sichern und viertens die projektierten Energieeinsparungen im Betrieb sichern (siehe dazu die Überschriften der Gruppen von Politikinstrumenten in Abbildung 4).

Im Policy Guide werden alle relevanten Informationen zusammengefasst. Hier finden sich:

- Ausführungen zum empfohlenen Politikpaket (im Internet unter: http://www.bigee.net/en/policy/guide/buildings/ recommended) schrittweise Herangehensweise ist für die Implementierung eines integrierten Designprozesses notwendig, um Energieeffizienz in neuen Bauprojekten und dem gesamten Gebäudesektor effektiv zu erreichen. Dieser integrierte Ansatz ist der Schlüssel zu einem komfortablen, wettbewerbsfähigen, energieeffizienten Gebäude sowie Baustein einer nachhaltigen Entwicklung.

Abbildung 5 vergleicht herkömmliche Gebäude mit drei Konzepten für energieeffiziente Gebäude. Die drei Konzepte sind Low-Energy Buildings, Ultra-Low-Energy Buildings und Zero- and Plus-Energy Buildings. 
Die Wahl zwischen diesen drei Konzepten ist nicht nur davon abhängig, wie ambitioniert ein Investor handeln will, sondern auch vom Verhältnis zwischen Kosten und Nutzen, der Qualität von Design und Bauausführung sowie Materialund Technologieverfügbarkeit in einem Land. Es wurden Konzepte für Wohnund Nichtwohngebäude, jeweils differenziert nach Neubauten und Bestandsgebäuden, entwickelt und diese werden weiter schrittweise veröffentlicht.

Der strategische Ansatz von bigEE, zunächst den Energieeinsatz maximal $\mathrm{zu}$ reduzieren und dann erneuerbare Energien einzusetzen, kann in den folgenden beiden Stufen zusammengefasst werden:

- Nachhaltige Reduktion des Verbrauchs der Gebäudeenergie von einem hohen $\mathrm{zu}$ einem niedrigen $\mathrm{Ni}$ veau durch integriertes angebots- und nachfrageseitiges Gebäudedesign (Low-Energy Building).

- Festlegung ambitionierterer Energiereduktionsstandards (Ultra-LowEnergy Building) und Implementierung von örtlicher erneuerbarer Energieversorgung, um nach Möglichkeit einen Energieüberschuss in der Jahresbilanz zu realisieren (Nearly-ZeroEnergy Building oder sogar Plus-Energy-Building).

Informationen zu den entwickelten Konzepten im Technologiebereich sind im Buildings Guide auf www.bigee.net zusammengefasst. Dieser ist wie folgt aufgebaut:

- Details zum integrierten Ansatz, der zur Realisierung jeder der drei Konzepte befolgt werden sollte, finden sich in der Sektion Strategic Approach (im Internet unter: http:// www.bigee.net/en/buildings/guide/residential/approach/ für Wohngebäude).

- Detaillierte Empfehlungen für Planer und Investoren, um diese Standards in den vier großen Klimazonen und für verschiedene Gebäudetypen zu erreichen, in der Sektion Recommendations (im Internet unter: http://www.bigee.net/ en/buildings/guide/residential/cases / - für Wohngebäude). Verschiedene Gebäudebeispiele von schon bestehenden Gebäuden der drei Typen in verschiedenen Klimazonen finden sich in der Sektion Building Examples (im Internet unter: http://www.bigee.net/en/buildings/guide/residential/examples / - für Wohngebäude). Hier finden sich auch Einzelheiten zur Konstruktion, den Gebäudesystemen, Energieeinsparungen und Wirtschaftlichkeit für diese Beispiele.

Weitere Hintergrundinformationen zum Thema Energieeffizienz in Gebäuden für die verschiedenen Bereiche sind in den jeweiligen Libraries der Guides zusammengetragen wor- den. Hier finden sich zum Beispiel auch Dokumente zum Thema Zusatznutzen von energieeffizienten Gebäuden und Geräten.

\section{Internationales Netzwerk}

Die Länderprojekte werden in Zusammenarbeit mit internationalen Projektpartnern durchgeführt. Es sollen sowohl private oder öffentliche Investoren, als auch politische Entscheidungsträger in Verwaltung oder Parlamenten adressiert werden. Die ersten Partnerländer von bigEE sind China, Indien und Südafrika. Dementsprechend baut das Projekt auf einem Netzwerk von Institutionen dieser Länder auf, welche Informationen sammeln, bearbeiten und aktiv verbreiten. Darüber hinaus kooperiert bigEE mit einigen internationalen Organisationen wie dem Umweltprogramm der Vereinten Nationen (UNEP), der International Partnership for Energy Efficiency Cooperation (IPEEC) und dem Global Buildings Performance Network (GBPN). Schließlich wird das Projekt bigEE von einem internationalen Beirat wissenschaftlich begleitet.

Dabei wurde deutlich, dass in den verschiedenen Ländern institutionelle Kapazitäten und die Datenlage sehr unterschiedlich sind sowie vielfach ein kohärenter Politikansatz fehlt. Für die Umsetzung von ganzheitlichen Politikpaketen, wie sie von bigEE vorgeschlagen werden, sind noch grundlegende Voraussetzungen in vielen Ländern zu schaffen. Besonders muss 
die Implementierung und Kontrolle beispielsweise von Baugesetzgebung und Gebäudestandards (Energy Building Codes und MEPS) sowie einer Zertifizierung sichergestellt und Fragen von Bewusstseinsbildung und Kompetenzaufbau der Akteure sowie einer Finanzierung in der Wertschöpfungskette adressiert werden.

Es lassen sich unterschiedliche Ansätze und Fortschritte auf dem Weg zu einer Dekarbonisierung des Gebäudesektors feststellen. Ihre bestehenden Ansätze sind oft nicht direkt mit den europäischen Ansätzen kompatibel. Hier stellt sich die Frage, in welchen Bereichen Wissenstransfer besonders nutzbar gemacht werden kann und wie Wissen den jeweiligen geografischen, politischen, sozialen und ökonomischen Gegebenheiten angepasst werden muss. Die zusätzlichen positiven Auswirkungen oder Zusatznutzen eines energetisch optimierten Neubaus oder von Bestandssanierung zum Beispiel für Armutsbekämpfung, Gesundheit und Komfort oder Produktivität müssen verdeutlicht werden, sind aber sozio-ökonomischen Rahmenbedingungen unterworfen. Wo der Zugang zu günstiger Energie und die Sicherung der Versorgung im Vordergrund stehen, sind andere Motivationsfaktoren zu adressieren.

Es wurde deutlich, dass eine reine Sammlung des bestehenden Wissens nicht ausreicht, sondern eine tiefergehende Erhebung und Aufbereitung der Daten zu Politikinstrumenten, Gebäudetechnologien und Geräten vonnöten war. Hohes Interesse besteht allseits an einem Austausch zu den deutschen und europäischen Ansätzen zur Gebäudeenergieeffizienz.

Aus dem Projekt haben sich weitergehende Formen der Zusammenarbeit mit Länderpartnern ergeben. So wurden für die Erarbeitung einer nationalen Gebäude-Roadmap für China auch Experten in Kooperation mit der Deutsche Gesellschaft für Internationale Zusammenarbeit konsultiert und so Beiträge für Roadshows und Trainings erarbeitet.

\section{Zukünftiger Forschungs- und Handlungsbedarf}

In den letzten Jahren wurde eine breite Basis an Informationen auf der Website www.bigee.net zusammengetragen und zeitgleich ein Netzwerk an Partnern aufgebaut. Beides gilt es in Zukunft auszubauen und zu verstetigen. Aktuelle Entwicklungen sollen auf der Plattform abgebildet werden. Dies betrifft auch neue Praxisbeispiele, die ergänzt werden sollen.

Zudem strebt bigEE an, die Verknüpfung mit anderen Plattformen wie dem Global Buildings Performance Network (GBPN), der Internationalen Energieagentur (IEA), der International Partnership für Energy Efficiency Cooperation (IPEEC) und der United for Efficiency Initiative der UNEP zu verbessern. So soll mittelfristig eine Plattform entstehen, die nicht nur Informationen zu Energieeffizienz in Gebäuden gesammelt zur Verfügung stellt, sondern gleichzeitig einen Ort bietet, um Partner, die in diesem Bereich arbeiten, miteinander in Kontakt zu bringen.
Nur gemeinsam kann die herausfordernde Aufgabe einer weltweiten Gebäudewende realisiert werden. Das dazu bestehende Wissen muss gebündelt werden und die Netzwerkpartner müssen zusammenarbeiten, damit die Potenziale für Energieeffizienz gehoben und die damit verbundenen ökologischen, sozialen wie ökonomischen Chancen im Gebäudesektor genutzt werden können.

\section{Literatur}

GEA, Global Energy Assessment (2012): Global Energy Assessment Toward a Sustainable Future. Cambridge (UK), Cambridge University Press. Cambridge.

IEA, International Energy Agency (2014): Energy Efficiency Market Report 2014. Paris.

Klinckenberg Consultants (2006): Better buildings through energy efficiency: A roadmap for Europe. Netherlands. Im Internet unter: www.bigee.net/ $\mathrm{s} / \mathrm{w} 5 \mathrm{~km} 5 \mathrm{~h}$

Sorrell, S. et al. (2004): The Economics of Energy Efficiency, Barriers to CostEffective Investment. Edward Elgar. Cheltenham.

Thomas, S. et al. (2013): Strategic policy packages to deliver energy efficiency in buildings - their international evidence. In: ECEEE 2013 Summer Study proceedings. S. 369-374.

Thomas, S. (2007): Aktivitäten der Energiewirtschaft zur Förderung der Energieeffizienz auf der Nachfrageseite in liberalisierten Strom- und Gasmärkten europäischer Staaten: Kriteriengestützter Vergleich der politischen Rahmenbedingungen. Kommunalwirtschaftliche Forschung und Praxis 13. Peter Lang. Frankfurt am Main.

Wuppertal Institut (2012a): How policies need to interact in packages. bigEE recommendations on which measures to combine for effective policy. Im Internet unter: http://www.bigee.net/media/filer_public/2013/11/28/ bigee_txt_0006_pg_how_policies_need_to_interact_2.pdf

Wuppertal Institut (2012b): A Strategic Approach enables Energy-Efficient Buildings. The key to energy efficiency. Im Internet unter: http://www.bigee.net/media/filer_public/2013/11/28/bigee_txt_0024_ bg_a_strategic_approach_enables_energy-efficient_buildings.pdf

\section{AUTOREN + KONTAKT} Döppersberg 19, 42103 Wuppertal. Tel.: +49 202 2492298, Döppersberg 19, 42103 Wuppertal. Tel.: +49 2022492268 ,
Jana Rasch ist wissenschaftliche Mitarbeiterin der Forschungsgruppe Energie-, Verkehrs- und Klimapolitik am Wuppertal Institut für Klima, Umwelt, Energie.

Wuppertal Institut für Klima, Umwelt, Energie, E-Mail: jana.rasch@wupperinst.org

Kilian Topp ist Projektleiter des Projekts bigEE und wissenschaftlicher Mitarbeiter der Forschungsgruppe Energie-, Verkehrs- und Klimapolitik am Wuppertal Institut für Klima, Umwelt, Energie.

Wuppertal Institut für Klima, Umwelt, Energie, E-Mail: kilian.topp@wupperinst.org

Dr. Stefan Thomas ist Leiter der Forschungsgruppe Energie-, Verkehrs- und Klimapolitik am Wuppertal Institut für Klima, Umwelt, Energie.

Wuppertal Institut für Klima, Umwelt, Energie,

Döppersberg 19, 42103 Wuppertal. Tel.: +49 202 2492143,
E-Mail: stefan.thomas@wupperinst.org

Döppersberg 19, 42103 Wuppertal. Tel.: +49 202 2492143,
E-Mail: stefan.thomas@wupperinst.org
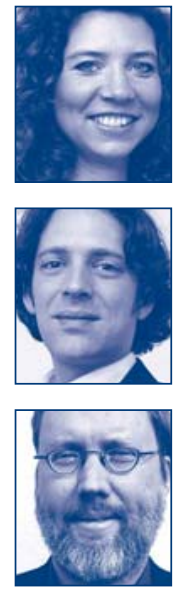